\title{
BMC Pediatrics reviewer acknowledgement 2014
}

Nawsheen Boodhun

\section{Contributing reviewers}

The editors of BMC Pediatrics would like to thank all our reviewers who have contributed to the journal in Volume 14 (2014).

Ellen Aartun

Denmark

Arturo Abdelnour

Costa Rica

Alaa Abd-Elsayed

Egypt

Yehia Abed

Palestinian Territory

Olumide Abiodun

Nigeria

Thomas Abramo

USA

Arianna Aceti

Italy

Eleri Adams

UK

Olusegun Adebami

Nigeria

Olufemi Adelowo

Nigeria

Miriam Adhikari

South Africa

Kingsley Agho

Australia

Kareem Airede

Nigeria

Akinyele Akinlade

Nigeria
Benjamin Albert

New Zealand

Luis M. Alegre

Spain

Ute Alexy

Germany

Khalid Alfaleh

Saudi Arabia

Sofiya Alhassan

USA

Paul Aljabar

UK

Bernt Alm

Sweden

Nicole Almenrader

Italy

Suzana Almoosawi

UK

Harith Al-Qazaz

Malaysia

Ghada Al-Rawahi

Canada

Jane Alsweiler

New Zealand

Teatske Altenburg

Netherlands

Jose Ramon Alvero Cruz

Spain
Luciano Alves Favorito

Brazil

Teresa Amaral

Portugal

Sergio Amarri

Italy

Geoffrey Ambler

Australia

Niels Holmark Andersen

Denmark

Satinder Aneja

India

Francois Angoulvant

France

Vincenzo Antona

Italy

Mitsuhiro Aoki

Japan

Christian Apfelbacher

Germany

Tomoki Arichi

UK

Tadashi Ariga

Japan

Emmanuel Arinaitwe

Uganda

Stephen Aronoff

USA

Correspondence: Nawsheen.Boodhun@biomedcentral.com

BioMed Central, Floor 6, 236 Gray's Inn Road, London WC1X 8HB, UK 
Pavai Arunachalam

India

Akira Ashida
Japan

Ambika Ashraf

USA

Greg Atkinson

UK

Stephanie Atkinson

Canada

Lamidi Isah Audu

Nigeria

Linda Aurpibul

Thailand

Inge Axelsson

Sweden

Eva Babusikova

Slovakia

Paluku Bahwere

Malawi

Gianluca Baio

UK

Muideen Bakare

Nigeria

Dian Baker

USA

Gareth Ball

UK

Quentin Ballouhey

France

Teresa Bandeira

Portugal

Robert Bandsma

Canada

Gerard Banez

USA

Sarfaraz Banglawala

Canada

Salvatore Barberi

Italy

Jane Barlow

UK

Michaela Barnard

UK
Jacqueline Barnes

UK

Benjamin Bar-Oz

Israel

Maru Barrera

Canada

Mauro Barros

Brazil

Michelle Barton-Forbes

Canada

Renato Bassan

Italy

Diego Garcia Bassani

Canada

Anna Basu

UK

Vijay Batra

India

Nerissa Bauer

USA

Consuelo Beck-Sague

USA

Lauren Becton

USA

Daniel Belsky

USA

Andrew Bennie

Australia

Nina Berentzen

Netherlands

Janet Berrington

UK

Gerard Berry

USA

Silvano Bertelloni

Italy

Liene Bervoets

Belgium

Marcel Betsch

USA

Ramesh Bhandari

Nepal

Jatinder Bhatia

USA
Sandeep Bhattacharya

India

Rahul Bhola

USA

Vinod Bhutani

USA

Sibhatu Biadgilign

Ethiopia

Sarah Biggs

Australia

Helen Binns

USA

Tracey Bjorkman

Australia

Clancy Blair

USA

Cynthia Blanco

USA

Jonathan Blau

USA

James Boardman

UK

Michael Boivin

USA

Srinivas Bolisetty

Australia

Attilio Boner

Italy

Maria Bonsignore

Italy

Erika Borkoles

Australia

Cynthia Boschi Pinto

Switzerland

Nancy Bostock

UK

Jérémie Botton

France

Marielle Karine Bouyou-Akotet

Gabon

Mauro Bozzola

Italy

Marek Brabec

Czech Republic 
Anneke Brand

Netherlands

Paul Brand

Netherlands

Rollin Brant

Canada

Hilde Kristin Brekke

Sweden

John F P Bridges

USA

Shobha Broor

India

Jason Brophy

Canada

Patrizia Bruzzi

Italy

Joseph Buckhalt

USA

Christoph Buhrer

Germany

James Bunn

UK

Ian Burgess

UK

Scott Burgess

Australia

Matthew Bush

USA

Alison Buttenheim

USA

Bert Callewaert

Belgium

Claudia Calogero

Italy

Rosa Calvo

Spain

Cecile Cames

France

Roque Cardona-Hernandez Spain

John Carlin

Australia

Magda Carneiro

Brazil
Genny Carrillo Zuniga

USA

Marco Caruselli

France

Sandra Carvalho-Bos

Portugal

Filip Cecka

Czech Republic

Carolina Chamorro-Vina

Canada

Alice Charach

Canada

Hassib Chehade

Switzerland

Wassim Chemaitilly

USA

Chung-Ming Chen

Taiwan

Shaojie Chen

China

Shawn Chen

Taiwan

Andreas Chiabi

Cameroon

Palma Chillón

Spain

Yitsiew Chin

Malaysia

Virginia Chomitz

USA

Voranush Chongsrisawat

Thailand

Hokyung Choung

South Korea

Rolando Cimaz

Italy

Emma Clark

UK

Cheril Clarson

Canada

Tine Dalsgaard Clausen

Denmark

Meryl Cohen

USA
Eyal Cohen

Canada

Conrad Cole

USA

R. Thomas Collins Ii

USA

Erica Cook

UK

Wouter Cools

Belgium

Peter Cooper

South Africa

Antonio Coppola

Italy

Giangennaro Coppola

Italy

E Corpeleijn

Netherlands

Iuri Corsini

Italy

Giuliana Cortese

Italy

Luciane Costa

Brazil

Malik Coulibaly

Burkina Faso

Charles Court-Brown

UK

Sherry Courtney

USA

Imelda Coyne

Ireland

Lisa Croen

USA

Estefania Custodio

Spain

Ron Dagan

Israel

Noémi Dahan-Oliel

Canada

Tuba Dal

Turkey

Marc Dalecki

Canada 
Theresa Dall Helth

Denmark

Diane Damiano

USA

Carlo Dani

Italy

M. Carolina Danovaro-Holliday USA

Peter Dargaville

Australia

Anne-Sophie Darlington

UK

Eugene Kofuor Maafo Darteh Ghana

Jai Das

Pakistan

Luca D'Ascanio

Italy

Reza Daugherty

USA

Sean Davies

USA

Andrew Day

Australia

Gianpaolo De Filippo

France

Elena De La Serna

Spain

Peter De Winter

Netherlands

Maartje De Wit

Netherlands

Iyabode Olabisi Dedeke

Nigeria

Seyed Mohsen Dehghani Iran

Mario D'Elios

Italy

Maurizio Delvecchio

Italy

Eugene Dempsey

Ireland

Ankita Desai

USA
Ulrich Desselberger

UK

Angelica Dessì

Italy

Kassahun Desta

Ethiopia

Anna Rita Di Biase

Italy

Graziella Di Grezia

Italy

Jose Luis Diaz-Rossello

Uruguay

Robert Digeronimo

USA

Sharmila Dissanaike

USA

Hazel Dockrell

UK

Nora Doering

Sweden

Monika Dos Santos

South Africa

Marjan Drukker

Netherlands

Jennifer Duchon

USA

Sourabh Dutta

India

Leigh Dyet

UK

Kevin Dysart

USA

Eric Dziuban

USA

Narelle Eather

Australia

Liza Edmonds

New Zealand

Matthias Egri-Okwaji

Nigeria

Christopher Bismarck Eke

Nigeria

Ashraf El Metwally

UK
Ann-Christin Eliasson

Sweden

Heather Elphick

UK

Nina Emaus

Norway

Stephen Eppes

USA

Omer Erdeve

Turkey

Melinda Erdos

Hungary

Esperanza Escortell

Spain

Christopher Imokhuede Esezobor

Nigeria

Sandrine Essouri

France

Irene Esteban-Cornejo

Spain

Charlotte Evans

UK

Jessica Ezri

Switzerland

Iretiola Fajolu

Nigeria

Giacomo Faldella

Italy

Kathryn Farrow

USA

Alan Farrow-Gillespie

USA

Moncef Feki

Tunisia

Juan Miguel Fernandez-Alvira

Spain

Bolanle Fetuga

Nigeria

Josep Figueras-Aloy

Spain

Peter Filan

Ireland

Martijn Finken

Netherlands 
Emily Finne

Germany

Saker Firas

USA

Marion Flechtner-Mors

Germany

Janice Fletcher

Australia

Sue Fletcher-Watson

UK

Cesar G Fontecha

Spain

Maria Forns

Spain

Terrence Forrester

Jamaica

Nathan Fosse

USA

Sotirios Fouzas

Greece

Lawrence Foweather

UK

Gustavo Fraga

Brazil

Adriana Franco

Brazil

Inge Franki

Belgium

David Freedman

USA

Helen Fryssira

Greece

Susan Fuchs

USA

Yuki Fujita

Japan

Ryuji Fukazawa

Japan

Jayne Fulkerson

USA

Daniel Fung

Singapore

Christoph Fusch

Canada
Dario Galante

Italy

Alexander Gallus

Australia

Stacey Galowitz

USA

Livia Garavelli

Italy

Anil Garg

UK

Orsolya Genzel

Germany

T Gheita

Egypt

Maria Gianni'

Italy

Peter Gerard Gibson

Australia

Stefania Giedrys-Kalemba

Poland

Cathleen Gillespie

USA

Flavio Giordano

Italy

John Giuliano

USA

Frances Glascoe

USA

Saralee Glasser

Israel

Sarah Goff

USA

Jeanne Goldberg

USA

David Goldfarb

Canada

Andrea Goldschmidt

USA

Vivianne Calheiros Chaves Gomes

Brazil

Antonia Gómez Conesa

Spain

Fangqi Gong

China
Timothy Goodacre

UK

Andy Goren

USA

Paul Govaert

Belgium

Monika Goyal

USA

Stephen Graham

Australia

Serge Grazioli

Switzerland

Christopher Greeley

USA

Siri Atma Greeley

USA

Ian Griffin

USA

Paul Gringras

UK

Floris Groenendaal

Netherlands

Giuseppe Grosso

Italy

Paulo Guerra

Brazil

Mintaze Gunel

Turkey

Ingibjorg Gunnarsdottir

Iceland

Rakesh Gupta

Nepal

Samir Gupta

UK

Sarika Gupta

India

Julie Gutman

USA

Gerda-Maria Haas

Germany

Karen Hacker

USA

Wendy Hall

Canada 
Michele Hamm

Canada

Branka Hanicar

Croatia

Iain Hargreaves

UK

Deirdre Harrington

UK

Jo Hart

UK

Annelies Hartman

Netherlands

Mohammad Hasan

Qatar

Ferdinand Haschke

Austria

Jean-Michel Hascoet

France

Amanda Hassinger

USA

Bryan Haughom

USA

Michael Hawkes

Canada

Shogo Hayashi

Japan

Nicole Hayde

USA

Meizi He

USA

Nongyue He

China

Xiangui He

China

Lana Hebden

Australia

Berit Heitmann

Denmark

Onno Helder

Netherlands

Krista Helleman

Canada

Nicholas Henschke

Germany
Michael Hermanussen

Germany

Kenneth Herrmann

USA

Egbert Herting

Germany

Ann Hickey

UK

Matthew Hicks

Canada

Takahiro Higashi

Japan

Marisa Hilliard

USA

Robert Hilliard

Canada

Trina Hinkley

Australia

Gerrit Hirschfeld

Germany

Jill Hnatiuk

Australia

Andrew Hodge

Australia

Falk Hoffmann

Germany

Rebecca Hommer

USA

Brian Hopkins

UK

Mary Horan

Ireland

Mina Hosseinipour

Malawi

Erin Howie

Australia

Yu-Shu Huang

Taiwan

Li Hui

China

Catherine Hunter

USA

Murad Husein

Canada
John Hutson

Australia

Arnold Huurnink

Netherlands

Bede Ibe

Nigeria

Aamer Imdad

USA

Marita Rohr Inglehart

USA

Moshe Ipp

Canada

Mary Irvine

USA

Iman Iskander

Egypt

Saurabh Jain

UK

Tim Jaspan

UK

Jennifer Margaret Jelsma

South Africa

Andreas Jenke

Germany

Ruth Jepson

UK

Milos Jesenak

Slovakia

Julie Jesson

France

Gerda Jimmy

Switzerland

Lars Jødal

Denmark

William Johnson

UK

Christine Jones

UK

Kelsey Jones

UK

Peter Jones

UK

Björn Jonsson

Sweden 
Shashank Joshi

India

Rumaya Juhari

Malaysia

Madarina Julia

Indonesia

Camille Jung

France

Joanna Jurewicz

Poland

Janine Jurkowski

USA

Masaharu Kagawa

Japan

Benjamin Kagina

South Africa

David Kalfa

USA

Satish Kalhan

USA

Hee Gyung Kang

South Korea

Henry J Kaplan

USA

Anne-Marie Kappelgaard

Denmark

Tonya Kara

New Zealand

Walter Karlen

Switzerland

Kianoush Kashani

USA

Lakshmi Katakam

USA

Anup Katheria

USA

Han Cg Kemper

Netherlands

Jorien Kerstjens

Netherlands

Anuradha Khadilkar India

Jaroslaw Kierkus

Poland
Jinkwan Kim

South Korea

Youngwon Kim

USA

Russell Kirby

USA

Gil Klinger

Israel

Martin Kneyber

Netherlands

Anders Koch

Denmark

Henrik Koehler

Germany

Anastasios Kollias

Greece

Joe Kossowsky

USA

Michael Kramer

Canada

Nancy Krebs

USA

Elena Krumova

Germany

Haytham Kubba

UK

Chandrakanta Kumar

India

Surender Kumar

India

Rashmi Kumar

India

Vishwajeet Kumar

India

Vasanth Kumar

USA

Ho-Chang Kuo

Taiwan

Ozgur Kurt

Turkey

Soyang Kwon

USA

Harrie Lafeber

Netherlands
Rajalakshmi Lakshman

UK

Veronica Lambert

Ireland

Steven Lamm

USA

Amy Lampard

USA

Claudio F. Lanata

Peru

Jason Lang

USA

Thorsten Langer

Germany

Malinee Laopaiboon

Thailand

Costas Laparidis

Greece

Alexandre Lapillonne

France

David P. Laplante

Canada

Antoinette Laskey

USA

Annie Lau

Australia

Elise Launay

France

Joshua Lawson

Canada

Kirsty Le Doare

South Africa

Nicole Le Saux

Canada

Wang-Tso Lee

Taiwan

Hung-Chang Lee

Taiwan

Pamela Lee

Vatican City State

Traci Leong

USA

Ting Fan Leung

China 
Ting Fan Leung

Hong Kong

Orly Levit

USA

Richard Lewanczuk

Canada

Albert Li

Hong Kong

Lun-De Liao

Singapore

Jean-Michel Liet

France

Choon Guan Lim

Singapore

Ming Lim

UK

Hung-Chih Lin

Taiwan

Ying-Jui Lin

Taiwan

Ornella Lincetto

Italy

Sergio Line

Brazil

Alexandre Linhares

Brazil

Yoav Littner

USA

Shuwei Liu

China

Rong Liu

China

Robert Locke

USA

Rakesh Lodha

India

Deirdre Logan

USA

Jamie Lohr

USA

Sebastian Loos

Germany

Armando Lorenzo

Canada
Linda Lowes

USA

Kelly Lowry

USA

Jeffrey Lu

USA

Laura Lucaccioni

Italy

Marta Lucchetta

Italy

Julie Lumeng

USA

Noni Macdonald

Canada

Aristides Machado-Rodrigues

Portugal

Michelle Macias

USA

Maciste Macias-Cervantes

Mexico

Andrea Maciel-Guerra

Brazil

Helen Mactier

UK

Jagmeet Madan

India

Agnieszka Madej-Pilarczyk

Poland

K S Madhusudhan

India

Jose Maia

Portugal

Nunzia Maiello

Italy

Imad Makhoul

Israel

Conor Mallucci

UK

Marisa Mancini

Brazil

Cedric Manlhiot

Canada

Jacob Manteuffel

USA
Paola Marchisio

Italy

Carole Marcus

USA

Dean Markic

Croatia

Jessica Markowitz

USA

Stephen Markwell

USA

Pedro Marques-Vidal

Switzerland

Stefano Marventano

Italy

Harald Matthes

Germany

Inas Mazen

Egypt

Mumtaz M Mazicioglu

Turkey

Graham Mccaffrey

Canada

H. David Mccarthy

UK

Eric Mccollum

Malawi

Nichola Mccullough

UK

Jenny Mcdonald

Australia

Eleanor Mcgee

UK

Meghan Mcgrady

USA

Patricia Mckane

USA

Ruth Mckee

UK

Stephen Edward Mcmillin USA

Catherine Mcneal

USA

Alan Mendelsohn

USA 
Jucille Meneses

Brazil

Christine Metz

USA

Fabrice Michel

France

Karen Mickle

Australia

Gregorio Paolo Milani

Italy

Christophe Milesi

France

Jonathan Miller

USA

Irene Milliken

UK

Matthieu Million

France

Jonathan Mintzer

USA

Rebecca Mitchell

Australia

Ron Mitchell

USA

Maurice B Mittelmark

Norway

Anup Mohta

India

Rachel Moon

USA

Laura Moore

USA

Dewton Moraes Vasconcelos

Brazil

Iris Morag

Israel

Fernando Moraga

Bangladesh

Alina Morawska

Australia

Henner Morbach

Germany

Philip Morgan

Australia
Prue Morgan

Australia

Ichiro Morioka

Japan

Michela Jane Angela Morleo

UK

Kevin Morris

UK

Rustin Morse

USA

Fabio Mosca

Italy

Anja Mowes

USA

Michael Msall

USA

Holger Muehlan

Germany

Noor Muhammad

Germany

Aleixo Muise

Canada

Luke Mullany

USA

Saul Mullen

Australia

Jan Müller

Germany

Caroline Mulvaney

UK

Antonella Muraro

Italy

Muhammad Umair Mushtaq

Pakistan

Victor Musiime

Uganda

Samridh Nagar

Australia

Koichi Nakanishi

Japan

Takahiro Nakayama

Japan

Gertrude Namazzi

Uganda
Nader Nassif

Italy

Nagato Natsume

Japan

Ellis Neufeld

USA

Mark Neuman

USA

Jason Newland

USA

Daniel Ng

Hong Kong

Glen Nielsen

Denmark

Signe Smith Nielsen

Denmark

Susan Niermeyer

USA

Fidelis Njokanma

Nigeria

Iona Novak

Australia

Anita Nucci

USA

Irena Nulman

Canada

Bright Nwaru

UK

Ju Lee Oei

Australia

Tinuade Ogunlesi

Nigeria

April Oh

USA

Bisola Ojikutu

USA

Ayodele Ojuawo

Nigeria

John Okeniyi

Nigeria

Julicristie Oliveira

Brazil

Laura Olivieri

USA 


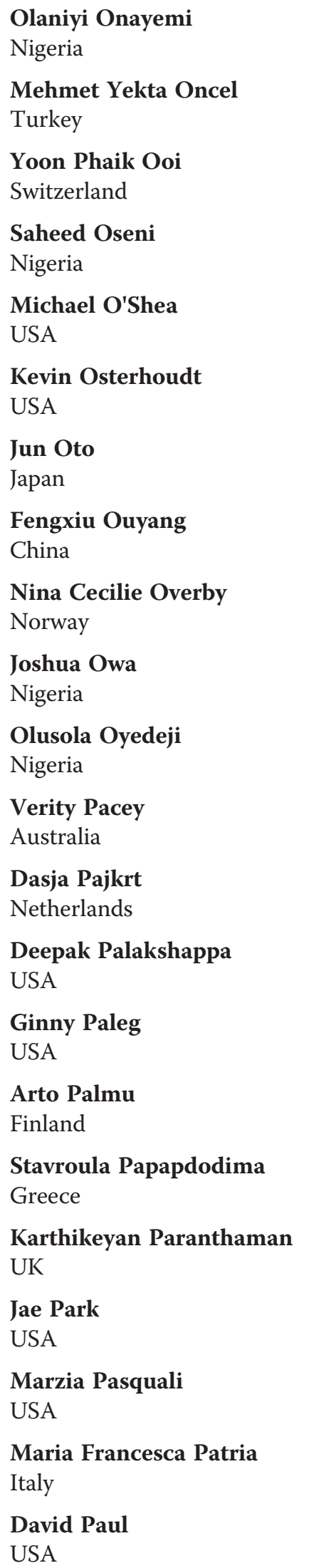

\begin{tabular}{|c|c|}
\hline $\begin{array}{l}\text { Malgorzata Paul } \\
\text { Poland }\end{array}$ & $\begin{array}{l}\text { Aziz Polat } \\
\text { Turkey }\end{array}$ \\
\hline $\begin{array}{l}\text { Stephen Pearlman } \\
\text { USA }\end{array}$ & $\begin{array}{l}\text { Alexey Polonikov } \\
\text { Russian Federation }\end{array}$ \\
\hline $\begin{array}{l}\text { Natalie Pearson } \\
\text { UK }\end{array}$ & $\begin{array}{l}\text { George Porter } \\
\text { USA }\end{array}$ \\
\hline $\begin{array}{l}\text { Lydia Pecker } \\
\text { USA }\end{array}$ & $\begin{array}{l}\text { Anna Price } \\
\text { Australia }\end{array}$ \\
\hline $\begin{array}{l}\text { Dino Pedrotti } \\
\text { Italy }\end{array}$ & $\begin{array}{l}\text { Joseph Price } \\
\text { USA }\end{array}$ \\
\hline $\begin{array}{l}\text { Giles Peek } \\
\text { UK }\end{array}$ & $\begin{array}{l}\text { Jochen Profit } \\
\text { USA }\end{array}$ \\
\hline $\begin{array}{l}\text { Suzanne Penfold } \\
\text { Slovakia }\end{array}$ & $\begin{array}{l}\text { Evan Propst } \\
\text { Canada }\end{array}$ \\
\hline $\begin{array}{l}\text { Suzanne Penfold } \\
\text { UK }\end{array}$ & $\begin{array}{l}\text { Jeremy Pryce } \\
\text { UK }\end{array}$ \\
\hline $\begin{array}{l}\text { Ji-Bin Peng } \\
\text { USA }\end{array}$ & $\begin{array}{l}\text { John William Lambert Puntis } \\
\text { UK }\end{array}$ \\
\hline $\begin{array}{l}\text { Supa Pengpid } \\
\text { Thailand }\end{array}$ & $\begin{array}{l}\text { Mikulas Pura } \\
\text { Slovakia }\end{array}$ \\
\hline $\begin{array}{l}\text { Marco Peres } \\
\text { Australia }\end{array}$ & $\begin{array}{l}\text { Niina Puustinen } \\
\text { Finland }\end{array}$ \\
\hline $\begin{array}{l}\text { Jeffrey Pernica } \\
\text { Canada }\end{array}$ & $\begin{array}{l}\text { Shamim Qazi } \\
\text { Switzerland }\end{array}$ \\
\hline $\begin{array}{l}\text { Roberto Perniola } \\
\text { Italy }\end{array}$ & $\begin{array}{l}\text { Liqian Qiu } \\
\text { China }\end{array}$ \\
\hline $\begin{array}{l}\text { Cynthia Peterson } \\
\text { Switzerland }\end{array}$ & $\begin{array}{l}\text { Jon Quach } \\
\text { Australia }\end{array}$ \\
\hline $\begin{array}{l}\text { Laetitia-Marie Petit } \\
\text { Switzerland }\end{array}$ & $\begin{array}{l}\text { José M Quintillá } \\
\text { Spain }\end{array}$ \\
\hline $\begin{array}{l}\text { Anne Pham-Huy } \\
\text { Canada }\end{array}$ & $\begin{array}{l}\text { Alejandro Quiroga } \\
\text { USA }\end{array}$ \\
\hline $\begin{array}{l}\text { Terri Pikora } \\
\text { Australia }\end{array}$ & $\begin{array}{l}\text { Omer Qutaiba B. Allela } \\
\text { Iraq }\end{array}$ \\
\hline $\begin{array}{l}\text { Daniele Piovani } \\
\text { Italy }\end{array}$ & $\begin{array}{l}\text { Jerilynn Radcliffe } \\
\text { USA }\end{array}$ \\
\hline $\begin{array}{l}\text { Maryam Piram } \\
\text { France }\end{array}$ & $\begin{array}{l}\text { Nedeljko Radlovic } \\
\text { Serbia }\end{array}$ \\
\hline $\begin{array}{l}\text { Paul L. Plener } \\
\text { Germany }\end{array}$ & $\begin{array}{l}\text { Melanie Rank } \\
\text { Germany }\end{array}$ \\
\hline $\begin{array}{l}\text { Christian Poets } \\
\text { Germany }\end{array}$ & $\begin{array}{l}\text { Susanna Ranta } \\
\text { Sweden }\end{array}$ \\
\hline $\begin{array}{l}\text { Marko Pokorn } \\
\text { Slovenia }\end{array}$ & $\begin{array}{l}\text { Rakesh Rao } \\
\text { USA }\end{array}$ \\
\hline
\end{tabular}


David Rappaport

USA

Sabrina Rasheed

Bangladesh

Maggie Redshaw

UK

Christopher Alan Reid

Australia

Aiguo Ren

China

James Renner

Nigeria

Bernhard Resch

Austria

Emrush Rexhaj

Switzerland

Corsino Rey

Spain

Zaccaria Ricci

Italy

Nicola Diane Ridgers

Australia

Marshall Riley

UK

Arieh Riskin

Israel

Calum Roberts

Australia

Joan Robinson

Canada

Elena Rodriguez-Rodriguez

Spain

Charles Christoph Roehr

UK

Martin Roland

UK

Nigel Rollins

South Africa

Diane Roscoe

Canada

Stephen Rose

Australia

Rebecca Rudd

USA
Franca Rusconi

Italy

Kelli Ryckman

USA

Carla Sabariego

Germany

Matthew Sabin

Australia

Manish Sadarangani

Canada

Raphael Saginur

Canada

Pilar Sainz De Baranda

Spain

Rie Sakai

USA

Leanne Sakzewski

Australia

Katherine Salamon

USA

Silvia Regina Saldiva

Brazil

Lucia Salesi

Italy

Shamiel Salie

South Africa

Vanessa Sanchez-Gistau

Spain

Francesca Santamaria

Italy

Sergio Santos

Brazil

Laura Sauve

Canada

Lindsay Schenkel

USA

Peter Schmidt

Australia

Lourdes Schnaas

Mexico

Sven M Schulzke

Switzerland

Jane Scott

Australia
Shannon Scott

Canada

André Seabra

Portugal

Jan Seghers

Belgium

Kris Sekar

USA

Ben Semmekrot

Netherlands

Moinak Sen Sarma

India

Jeremiah Seni

Tanzania

Ali Sepahdari

USA

Mary Serdula

USA

Raju Shah

India

Sameena Shah

Pakistan

Ketan Shankardass

Canada

Iman Sharif

USA

Eesha Sharma

India

Yiping Shen

USA

Xiaoyang Sheng

China

Patricia Shewokis

USA

Ling Shi

USA

Zhongjie Shi

USA

Jung Yeon Shim

South Korea

Masaki Shimizu

Japan

Kevin Short

USA 
Shyh-Dar Shyur

Taiwan

Loreana Silveira

Brazil

Aryeh Simmonds

Israel

Ajay Sinha

UK

Susan Sisson

USA

Seter Siziya

Zambia

Joseph Skelton

USA

Marianne Skreden

Norway

Linda Slack-Smith

Australia

Tina Slusher

Nigeria

Françoise Smets

Belgium

Claire Smith

UK

Lucy Smith

UK

Joern Soltau

USA

Madhuri Sopirala

USA

Maroje Soric

Croatia

Rick Speare

Australia

Carsten Speckmann

Germany

Jan Sperhake

Germany

Claudio Spinelli

Italy

Jane Squires

USA

Vijay Srinivasan

USA
Keith St Lawrence

Canada

Philippe Steenhout

Switzerland

John Stefano

USA

Sarah Stewart-Brown

UK

Blanka Stiburkova

Czech Republic

John F. Stins

Netherlands

Chris Stockmann

USA

Jana Strahler

Germany

Robyn Stremler

Canada

Wendy Sturtz

USA

Adewale Okanlawon Sule-Odu

Nigeria

Kevin Sullivan

USA

Carolyn Summerbell

UK

Xin Sun

China

Sharon Sung

Singapore

Jc Suris

Switzerland

Gordana Susic

Serbia

Graeme Suthers

Australia

Elisabeth Svensson

Sweden

Meenakshi Swaminathan

India

David Sweet

UK

Dorota Szostak-Wegierek

Poland
Taro Takeshima

Japan

Ajay Talati

USA

Alison Talbert

Kenya

Pooja Tandon

USA

Patrick Tang

Canada

Jose Tapia

Chile

Kimberly Tartaglia

USA

Richard Telford

Australia

Roger Thomas

Canada

Pam Thomason

Australia

Dana Thompson

USA

Michael Thompson

USA

Dick Tibboel

Netherlands

Alison Tigg

Australia

Janice Tijssen

Canada

Peter Tilley

Canada

Ariel Toloza

Argentina

Olukemi Tongo

Nigeria

Karina Top

Canada

Susan B Torrey

USA

Antonella Tosti

USA

Leonardo Trasande USA 
Victoria Trenchs

Spain

Antonia Trichopoulou

Greece

Chang-Yong Tsao

USA

Takeshi Tsutsumi

Japan

Michael Tunik

USA

Jos Twisk

Netherlands

Agozie Ubesie

Nigeria

Raphael Udassin

Israel

Holm Uhlig

UK

Nicola Ullmann

Italy

Michael Urschitz

Germany

Nestor Vain

Argentina

Christina Valentine

USA

Monique Van De Lagemaat

Netherlands

Hubertus Van Hedel

Switzerland

Wendy Van Lippevelde

Belgium

Femke Van Nassau

Netherlands

Frits Van Rhee

USA

Diny Van Zoeren-Grobben

Netherlands

Jillon Vander Wal

USA

Leigh Vanderloo

Canada

Patrick Vandevoorde

Belgium
George Vaos

Greece

Ivan Varga

Slovakia

Atul Vats

USA

Timothy Vece

USA

Pierangelo Veggiotti

Italy

Haritha Vellanki

USA

Maximo Vento

Spain

Charles Verge

Australia

Nishant Verma

India

Rita Verma

USA

Ritu Verma

USA

Ruud Vermeulen

Netherlands

Sunil Vernekar

India

Vanessa Ann Vigilante

USA

Jeffrey M Vinocur

USA

Tommy Visscher

Netherlands

Carl Von Baeyer

Canada

Sarah Von Spiczak

Germany

Wieger Voskuijl

Malawi

Amos Vromen

Israel

Anne Vuillemin

France

Gordan Vujanic

UK
Julian Vyas

New Zealand

Julee Waldrop

USA

Andrew Walley

UK

Guoying Wang

USA

Martin Ward Platt

UK

Klaas Wardenaar

Netherlands

Malgorzata Wasniewska

Italy

Andrea Waylen

UK

Ashley Weaver

USA

Patricia Weng

USA

Karla Wentzel

Canada

Fred Were

Kenya

Benjamin Wheeler

New Zealand

Elspeth Whitby

UK

Chris White

Australia

Andrea Wickremasinghe

USA

Kurt Widhalm

Austria

Frank Wieringa

France

Bridget Wilcken

Australia

Beth Wildman

USA

James Wiley

USA

Suzanne Willard

USA 


\begin{tabular}{|c|c|c|}
\hline Paige Williams & Charlotte Wright & Dp Zhao \\
\hline USA & UK & China \\
\hline Jennie Wilson & Julie Wright & Lili Zhao \\
\hline UK & USA & USA \\
\hline Kathleen Wilson & Robert Wright & Guangwei Zhou \\
\hline USA & USA & USA \\
\hline Deanne Wilson-Costello & Rebecca Wyse & Ying Zhou \\
\hline USA & Australia & USA \\
\hline Esko Wiltshire & Yao-Jong Yang & Jiajun Zhu \\
\hline New Zealand & Taiwan & China \\
\hline Yk Wing & Allen Eng Juh Yeoh & Vahid Ziaee \\
\hline Hong Kong & Singapore & Iran \\
\hline Corinna Winter & Ediz Yesilkaya & Joseph Zickafoose \\
\hline Germany & Turkey & USA \\
\hline David Wirtschafter & Bridget Young & Jerry Zimmerman \\
\hline USA & UK & USA \\
\hline Cicek Wöber-Bingöl & Jeanine Young & Maddalena Zippi \\
\hline Austria & Australia & Italy \\
\hline Cicek Wöber-Bingöl & Zhangbin Yu & Alvin Zipursky \\
\hline Germany & China & Canada \\
\hline Joseph Wolfsdorf & Justin Zachariah & Elizabeta Zisovska \\
\hline USA & USA & Macedonia \\
\hline Katja Wolthers & Samuel Zangen & Anna Zolotnitskaya \\
\hline Netherlands & Israel & USA \\
\hline Craig Wong & Huan Zeng & Gian Vincenzo Zuccotti \\
\hline USA & China & Italy \\
\hline Melanie Wong & Jihui Zhang & Rose Zulliger \\
\hline Australia & Hong Kong & USA \\
\hline Kyung In Woo & Nan Zhang & Jill Zwicker \\
\hline South Korea & UK & Canada \\
\hline Jayne Woodside & Linjie Zhang & \\
\hline UK & Brazil & \\
\hline
\end{tabular}

